\title{
Generalizing over encounters: Statistical and theoretical considerations
}

\author{
Dale J. Barr \\ University of Glasgow
}

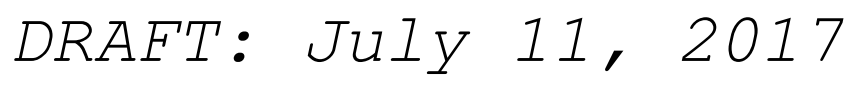

How do we evaluate evidence for the generality of phenomena in psycholinguistics and related fields? For decades, researchers have recognized the importance of treating not only participants, but also stimuli, as sampled rather than fixed (Clark, 1973). It is only by taking both subject and stimulus populations into account during analysis - and critically, doing so simultaneously — that claims about psycholinguistic phenomena are supported in their full generality. In this chapter, I argue that simultaneous by-subject and by-item analyses is a special (albeit common) case of a more general problem of generalizing over particular types of encounters, defined in terms of the sampled units involved in the situation and the connections between them. This approach is more general inasmuch as it encompasses the traditional two-party encounters between subjects and stimulus materials but also handles two-party dyadic communicative encounters, or even three-party encounters involving participants making judgments about stimuli that were produced by participants in response to other stimuli. I argue that the goal of analysis is to make claims that are maximally likely to generalize to new encounters of the same type, and propose that the relevant encounter types involved in an experiment can be identified by thinking about the boundaries of what would constitute a legitimate 'replication' of that experiment. These ideas have important implications far beyond psycholinguistics; indeed, nearly all areas of psychology and neuroscience make claims that are intended to generalize to new encounters, although it is only psycholinguistics that consistently uses the statistical apparatus required to support such claims.

This chapter is organized as follows. In the first part, I review the historical development of these issues within the field of psycholinguistics. Next, in what is the main contribution of this chapter, I propose a more general perspective on the issue of simultaneous generalization by developing the notion of encounter types and argue that simultaneous generalization over subjects and items is a special case of this more general problem. In the third and final section, I discuss the consequences of neglecting this perspective, address some common misconceptions, and consider the technical challenges involved in statistical generalization over encounters.

\section{Background: The 'language-as-fixed-effect' fallacy}

Since Coleman (1964) and Clark (1973), psycholinguists have typically analyzed data treating both participants and stimuli as random factors. Clark, like Coleman before him, pointed out that researchers who use language materials (such as words or sentences) as stimuli obviously do not sample exhaustively from the population of possible stimuli, but use only a subset of materials that they present repeatedly to different participants. Because people's responses to particular stimuli will vary systematically (e.g., some words will be more memorable or easier to recognize than others), the various responses to a given stimulus are not independent. If this variation is not accounted for in the inferential analysis, it will tend to inflate the false positive rate. Clark (1973) 
proposed an ANOVA-based inferential approach which took by-subject and by-item variation into account through statistics such as $F^{\prime}$ or min- $F^{\prime}$. Although there was initially some controversy about the need for by-item analysis (see Cohen, 1976; Smith, 1976; Wike \& Church, 1976), psycholinguists quickly adopted Clark's proposals, and statistics such as min- $F^{\prime}$ became a regular feature of psycholinguistics papers.

Clark's advice was heeded in spirit, but within a decade, researchers were more often doing a kind of analysis that he had advised against - separately conducted by-subject and by-item analyses, with by-subject analyses reported as $F_{1}$ (or $t_{1}$ ) and by-item analyses reported as $F_{2}$ (or $t_{2}$ ) - and not the simultaneous analyses embodied in the single statistic min- $F^{\prime}$. It is unclear why this happened, although researchers probably abandoned min- $F^{\prime}$ upon realizing that it is overly conservative in certain cases (Forster \& Dickinson, 1976). In the separate analysis version (henceforth $F_{1} \times F_{2}$ ), researchers would conclude that an effect was significant only if both $F_{1}$ and $F_{2}$ were significant. At least that was the idea; in practice, more weight was given to the by-subject analysis, and authors, editors, and reviewers would sometimes let a nonsignificant $F$ statistic slide if it was from the byitem analysis. But as Clark pointed out, the $F_{1} \times F_{2}$ analysis is invalid, because the test for each sampling unit is influenced by variation from the sampling unit neglected in that analysis. Because of this, both $F_{1}$ and $F_{2}$ can be significant individually even when the null hypothesis is true, with the significance of $F_{1}$ driven by neglected by-item variation and that of $F_{2}$ driven by neglected bysubject variation. And despite additional attempts to call attention to the invalidity of $F_{1} \times F_{2}$ in the late 1990s (Raaijmakers, Schrijnemakers, \& Gremmen, 1999) as well as more recently (Barr, Levy, Scheepers, \& Tily, 2013), to this day, $F_{1} \times F_{2}$ remains the most common approach among ANOVA users in psycholinguistics.

Use of ANOVA, however, rapidly diminished in psycholinguistics once linear mixed-effects models (LMEMs) appeared on the scene, especially after an influential paper by Baayen, Davidson, and Bates (2008) introduced psycholinguists to the lme4 package for R (Bates, Mächler, Bolker, \& Walker, 2015) and showed how it could be used to support simultaneous generalization. The idea of using LMEMs in this way had been suggested before (Locker, Hoffman, \& Bovaird, 2007; Quené \& van den Bergh, 2004), but it was the wide availability of the lme4 package and tutorial examples in Baayen et al. (2008) that accelerated its uptake. LMEMs were attractive not only because they solved the problem of simultaneous generalization, but also because they made it easier to model effects of continuous covariates (e.g., time, age, word frequency) while simultaneously offering greater flexibility to adjust distributional assumptions to the type of data being analyzed (e.g., using binomial distributions for dichotomous data or Poisson distributions for count data).

Psycholinguists took up LMEMs with enthusiasm, but many of the early papers did not heed warnings about the need to carefully consider how sampling unit variation should be structured in the model (Barr et al., 2013).

Sampling units such as subjects or items introduce variation that can be associated with different components of a linear model, and this variation is typically divided into random intercept and random slope variance. Many researchers who were new to LMEMs were fitting models that accounted for random intercept variation, but did not worry about random slope variation. Random intercept variance is the variance is associated with the intercept term of a linear model. Much like a political poll by a particular organization can have a "house effect" - a consistent bias toward a particular party or candidate - a given sampling unit can also push a response variable in a consistent direction by a consistent amount. Certain subjects will be systematically less accurate or slower in their responses than others across trials. Likewise, certain stimuli will occasion greater delay or higher rates of errors than others. But sampling units can vary not just in this kind of "house effect" but can also vary in their sensitivity to experimental manipulations, introducing random slope variation. Some participants will more sensitive than others to the difference between concrete and abstract words, or more impaired by a cognitive load manipulation. The variance 
associated with both of these types of "house effects" should be considered when estimating a mixed-effects model.

Of the early psycholinguistics papers using mixed-effects modeling, many-perhaps even mostestimated random intercept variation but ignored random slope variation, with potentially dire inferential consequences. Use of random-intercept-only models was also rampant among early adopters of mixed-effects models in fields outside of psychology (Schielzeth \& Forstmeier, 2009). Monte Carlo simulations suggested that applying random-intercept only models to the very common experimental design in which a factor was manipulated within subjects and within items produced higher false positive rates than ANOVA performed on subjects alone. Barr et al. (2013) therefore encouraged researchers to "keep it maximal": identify those potential sources of sampling unit variation that are identifiable (i.e., that can be distinguished from residual error), and ensure they are accounted for in the model. Their simulations demonstrated that under a wide range of scenarios, a model with maximal random effects structure showed the best performance in terms of both Type I error and power. Indeed, the power of maximal models was much greater than min- $F^{\prime}$ and comparable to that obtained using the (invalid but widely used) ANOVA-based $F_{1} \times F_{2}$ approach. While Barr et al. (2013) argued that judgments about random effects should be driven by the experimental design, others have suggested letting the data determine the random effects structure (Matuschek, Kliegl, Vasishth, Baayen, \& Bates, 2017). Both approaches recognize that considering both random intercept and random slope variance is essential for sound inference.

\section{A broader perspective: Generalizing over encounters}

Although Clark's points about the conditions necessary for full generalization are applicable to many research areas, his framing of the problem in terms of language stimuli may have limited the uptake of these ideas outside of psycholinguistics. Also, discussion of the problem in terms of separate subject and stimulus populations may have contributed to the misconception that the problem is solved by separate by-subject and by-item analyses. In this section, I argue that the proper unit of analysis is the encounter: we seek to say something general about what happens when a particular type of sampling unit (e.g., subject) interacts with another type of sampling unit (e.g., stimulus). This perspective locates the traditional case of simultaneous generalization to subjects and items as one point in a larger space of theoretical possibilities. I suggest that thinking about statistical generalization in this way makes it easier to translate a study's design into an appropriate analysis plan.

Many claims in psycholinguistics are inherently claims about encounters - neither claims about people nor claims about language considered separately—but claims about particular types of people producing or understanding particular types of language in particular types of contexts. For instance, a claim that speakers are primed more by low- than high- frequency syntactic structures is a claim about what happens when a particular type of sampling unit (speaker) encounters another particular type of sampling unit (sentence); we say they are sampled because the claim is intended to apply to the effect of any arbitrary sentence structure on any arbitrary speaker, not just the sentences and speakers used in the experiment. Similarly, the claim that certain hormones modulate the level of attraction female participants feel toward dominant male faces is a claim about an encounter between an arbitrarily chosen female participant and an arbitrarily chosen male face. In short, in experiments involving samples of subjects and stimuli, it is not so much that we wish to generalize 'simultaneously' to multiple target populations, but that we wish to say something about the single population of possible encounters between the two types of units.

I believe there are certain benefits to this way of viewing the problem of statistical generalization. First, by emphasizing generalization to events rather than generalization to things, it becomes clearer that the type of entity involved in the event - people, pictures, words, voices, birds - is not 
important; what is important is the fact that they are sampled as well as the manner in which they relate to one other in the study. There is no good reason why psycholinguists routinely treat their stimuli as random while face perception researchers routinely treat their stimuli as fixed; it is merely different analytical conventions for different fields. It doesn't matter what is being measured (behavior or brain responses) or even whether the study involves human populations at all. A researcher who studies birds' reactions to bird songs would aim to say something general about how a certain type of bird responds to a certain type of song, and unless her sampling procedure exhausts the possible combinations of these types of birds and types of songs, she would need to treat both birds and songs as random in her analysis. Other fields need to learn the lessons of psycholinguistics, and are more likely to learn these lessons if they are framed in terms of the general properties of events, not of things.

Second, the notion of an encounter provides a single target population for inferential claims: a population of events of a given type involving certain sampling units organized in a certain way. It is easier to think about scientific claims in terms of a single target population rather than in terms of multiple target populations simultaneously. For instance, claims about the procedures involved in lexical access are about people perceiving and reacting to words, not claims about people and claims about words. Viewed thus, becomes much clearer why separate by-subject and by-item analyses are inappropriate. To say something general about a type of event, one must be able to separate out general characteristics of the encounter from the idiosyncratic properties of the units (subjects or items) involved. Inference at the subject (or item) level alone, in which trials are aggregated together to calculate a set of participant (or item) means, inevitably confounds these sources of variation, and because they cannot be pulled apart, inference becomes bound to the specific units that are neglected in the analysis.

Thinking about generalization in terms of encounters may also aid interpretation of fixed effects in a mixed-effects model. An encounter is essentially an idealized typical trial in an experiment. Thinking about what units are involved in a given trial and whether they should be treated as sampled or fixed is key to identifying the appropriate analysis. And the fixed effects estimates from a linear-mixed effects model with crossed random effects are directly interpretable in terms of the basic encounter being studied: they can be viewed as the model's best guess at how a typical participant responds to a typical item on a typical trial. Thus, inferences that models provide about these parameter estimates are, in fact, inferences about a target population of encounters.

Finally, thinking about generalization to new events may aid in appreciating the limits on the generality of a study's claims. For instance, many studies of dialogue often involve a listener who interprets expressions from a speaker, while some aspect of the listener's behavior (e.g., eye gaze) is measured. The fundamental event in such studies involves three units: a speaker, a listener, and a linguistic expression. In principle, the researcher would like to make general claims about events involving an arbitrary listener interpreting a particular type of expression produced by an arbitrary speaker. However, for reasons of convenience, such studies often will use a fixed speaker for all participants in the experiment while at the same time sampling listeners and expressions. There will be idiosyncratic characteristics of this fixed unit (e.g., the speaker is male or female, enthusiastic or disengaged, has a dialect reflecting a particular socioeconomic status) that can impact how listeners respond to his or her expressions, and that may even impact different kinds of listeners in different ways. At one extreme, the experiment could be even be considered as a case study of this particular speaker. Making fully general claims would require sampling not just listeners and expressions but also speakers. If this is impractical, the researcher would need to provide arguments as to why this speaker should be treated as representative. But one seldom sees discussion of such limitations in papers on dialogue, possibly because of the focus on generalizing things (subjects and stimuli) rather to event types. 


\section{Types of encounters}

The problem of generalizing over participants and stimuli is just a special case (albeit, an extremely common one) of the more general problem of generalizing over encounters. In this section, we consider how this perspective can be extended to different kinds of encounters, involving different types of units, different numbers of these types (e.g., three-party encounters instead of two-party encounters), and different organizations of the units in the encounter.

Most obviously, encounters can involve stimuli of a non-linguistic nature. To choose but one example, studies of face perception investigate how particular classes of people judge particular classes of faces. It may be of interest, for example, to examine how dominance characteristics in male faces interact with a female respondent's hormonal profile to influence attractiveness judgments. In such a study, both female participants and male faces should be considered sampled; however, most face perception studies ignore the sampled nature of materials, but without circumscribing their claims to the particular sample of stimuli. As a consequence, claims may generalize to new female respondents encountering the same male faces, but not to new samples of male faces. The same goes for studies in social psychology, where stimuli are often chosen to represent people or objects of social relevance (Judd, Westfall, \& Kenny, 2012). For instance, studies looking at the detection of vocal signals of competence or trustworthiness must account for the sampled nature of the voices used in the experiment.

It should also be fairly obvious that the problem of generalizing over encounters is completely independent of the type of dependent measure involved in a study. Given this, it is perplexing that neuroimaging studies - even neuroimaging studies carried out by psycholinguists - rarely take stimulus variation into account, despite calls to do so (Bedny, Aguirre, \& Thompson-Schill, 2007). But even the few studies that implement item analyses often do so inappropriately, pursuing separate rather than simultaneous generalization. One barrier might be the complexity of estimating many mixed effects models over many variables (EEG sensors or voxels), but recent work by Westfall, Nichols, and Yarkoni (2016) shows that this is technically feasible, and improves the quality of inference.

Sometimes the encounters one wishes to generalize over involve people reacting to particular types of people rather than particular types of stimuli. A recent study that received much media attention reported gender bias in students' evaluations of instructors, with male instructors rated as better than female instructors (MacNell, Driscoll, \& Hunt, 2015). Students interacted online with either a male or female instructor and later rated aspects of the instructor's performance. The online nature of the interaction made it possible for experimenters to manipulate students' perceptions of the gender of the instructor independently of the instructor's actual gender. This was accomplished by having one male and one female instructor lead a discussion group either under their own identity or while pretending to be the instructor of the opposite gender. The study found that students gave lower ratings when they perceived the instructor to be female than when they perceived the instructor as male. While this finding is interesting, the study's use of only two instructors makes it essentially a case study of the performances of these particular instructors. It would require multiple instructors of each gender to have any confidence in its generality.

Certain types of studies involve three-party encounters, such as when pairs of participants communicate about pictorial stimuli (Garrod, Fay, Lee, Oberlander, \& MacLeod, 2007). Or, a threeparty encounter might involve a single participant responding to stimuli from two different populations, such as in a priming study where a picture is flashed on the screen prior to the presentation of a probe word. In the latter case, whether the stimuli should be treated in the analysis as separate random factors or as a single random factor representing each pair would depend upon whether each individual stimulus is ever used outside of a single pairing. If each stimulus is only used within a pair, it wouldn't be possible to statistically distinguish effects of the pairing from the 
individual effects of each picture and word, and so the pairs of stimuli should be treated as a single factor.

A final, interesting case is offered by studies that use human subjects as 'measuring devices' to assess perceptual constructs whose physical characteristics are not well-established or are subjective and therefore difficult to measure. For example, in a study on face perception, a set of participants might provide attractiveness ratings for a set of faces that are then presented to another set of participants. In psycholinguistics, this kind of situation arises in the AXB discrimination task, in which participants rate whether a spoken word token $\mathrm{X}$ is more similar to token $\mathrm{A}$ or token $\mathrm{B}$. For instance, Pardo (2006) used an AXB task to examine phonetic convergence between pairs of interacting speakers. Encounters in this study involved multiple sampling units: the pairs of participants whose interaction gave rise to individual speech tokens, and the raters who later assessed the similarities of these tokens. What is interesting about this type of study is that it involves subjects from one sample making judgments about the behaviors produced by another set of subjects. Indeed, there is also a fourth potential sampling unit involved: the particular word the speaker uttered (a landmark on a map). Although the real target of generalization is the conversational encounter between the interacting participants, the measurements of this encounter will also be influenced by characteristics of particular set of raters that were sampled. Unfortunately, we currently know little about the inferential consequences of treating raters as fixed in these types of studies.

In sum, human subjects responding to stimuli is but one of a large variety of encounter types that can arise in different areas of psychology and neuroscience. Given this variety, how does one recognize the target population of encounters to which findings should be generalized?

Characterizing the encounter type is not difficult because it usually follows directly from the main claim the study is seeking to support, which in turn is often realized in the structure of a typical trial within the experiment. But what can be more difficult to identify is which aspects of an encounter should be treated as fixed and which should be treated as random.

This latter judgment can be aided by thinking in terms of replication: which aspects of the encounter are essential and should be preserved in a direct replication, and which should be allowed to vary? Usually the essential part involves the structure of the encounter and the types of entities it involves, and the arbitrary part involves the identities of these types as they are realized through the set of sampled subjects and/or stimuli. For instance, when considering a replication of the study on instructor gender bias mentioned above (MacNell et al., 2015), it becomes clear that the particular identities of the instructors are nonessential to the study's claims, as the claims are intended to apply generally to any male or female instructors. Thus, a more robustly generalizable test of this hypothesis would have required a sample of male and female instructors in addition to the obtained sample of students, and without this additional sampling claims about generality should be tempered.

We would not be inclined to count a study as a 'replication' if it was conducted on the exact same participants as the original, and not just because there is no magic way to wipe away subjects' memories of their experience in the original before participating in the replication. A good replication should sample new 'levels' of the subject factor to help minimize the possibility that the original result is due to sampling error. In contrast, researchers regularly view it as desireable and even essential to use the exact same set of stimulus materials in a replication as in an original study, often counting the use of the same stimuli as a criterion for being a 'direct' replication. However, this sacrifices generalizability for the sake of fidelity; if the claims are about the class of stimuli involved in the encounter, and not the particular stimuli involved, then a direct replication should involve new stimuli as well as new participants (Westfall, Judd, \& Kenny, 2015). More precisely, it should involve new encounters among new sampling units from the constituent populations. 


\section{Statistical issues in generalizing over encounters}

In this section, I consider the issue of how to specify a statistical model so that claims based on the model will best generalize to a new sample from a target population of encounters. The section begins by presenting the general problem that is created by crossing sampling units in a series of encounters: namely, each repeated observation on sampled units erroneously treated as fixed amplifies the impact of sampling error on interference. Along the way, I discuss how to determine which random effects to include in a model, and the consequences of treating units as fixed rather than random. I close by discussing a few cases that are commonly misconceived as requiring only by-subject analysis.

\section{Repetition amplifies the impact of sampling error}

A sample from a population, being a subset of that population, is unlikely to be completely representative of the population as a whole. The extent to which it is representative depends on the size of the sample relative to the population, along with the magnitude of variation in the population. Proportionally small samples will tend to be less representative on average than larger samples. Even fixing the sample size, departures from the target population will differ from sample to sample.

This issue of sampling error is well-known and its implications are fairly obvious. However, what is often under-appreciated is that when treating sampled units (subjects, stimuli) as fixed in the analysis, each repeated measurement on these same sampled units amplifies the distorting influence of sampling error. In a design with crossed random factors of subjects and stimuli, each new subject typically implies a new repetition of measurements across the same set of stimuli. Likewise, each additional stimulus implies a repetition of measurement across the same set of subjects. In an analysis where subjects are considered as random but stimuli are neglected, increasing the bysubject sample size will tend to increase the distorting influence of the sampling error associated with stimuli. So long as a sampling unit in the encounter is neglected, increasing the sample size of any other considered unit may actually increase the false positive rate and thus decrease the generality of any claims with regard to the target population of encounters.

Researchers who conduct large scale replication studies involving many subjects at many different sites should take heed of this. While the ever-increasing ease of data collection through online methods would, at first blush, seem to a boon for generalizability and replicability, large samples with the wrong analysis can paradoxically lead to greater rates of false positive findings.

A simple Monte Carlo simulation demonstrates this phenomenon. The simulation assumes there is variation in sensitivity to a manipulation over subjects and items, but no consistent overall effect. Thus, any significant result is a false positive. We kept the magnitude of this variation fixed while varying the size of the stimulus sample (20 versus 40) and the size of the subject sample (from 20 to 500 in steps of 60). ${ }^{1}$ Figure 1 presents the results where each analysis treated subjects as random but ignored stimulus variation. The false positive rate was clearly unacceptably large at all sample sizes, but increased dramatically as the subject sample size increased, approaching .6 for the largest samples, nearly four times the rate observed for the smallest samples.

1 The code for the simulation can be found at https://gist.github.com/dalejbarr/8f6adabdbf2ab9b3458cf51c7bfee484. 


\section{Type I error when stimuli treated as fixed not random}

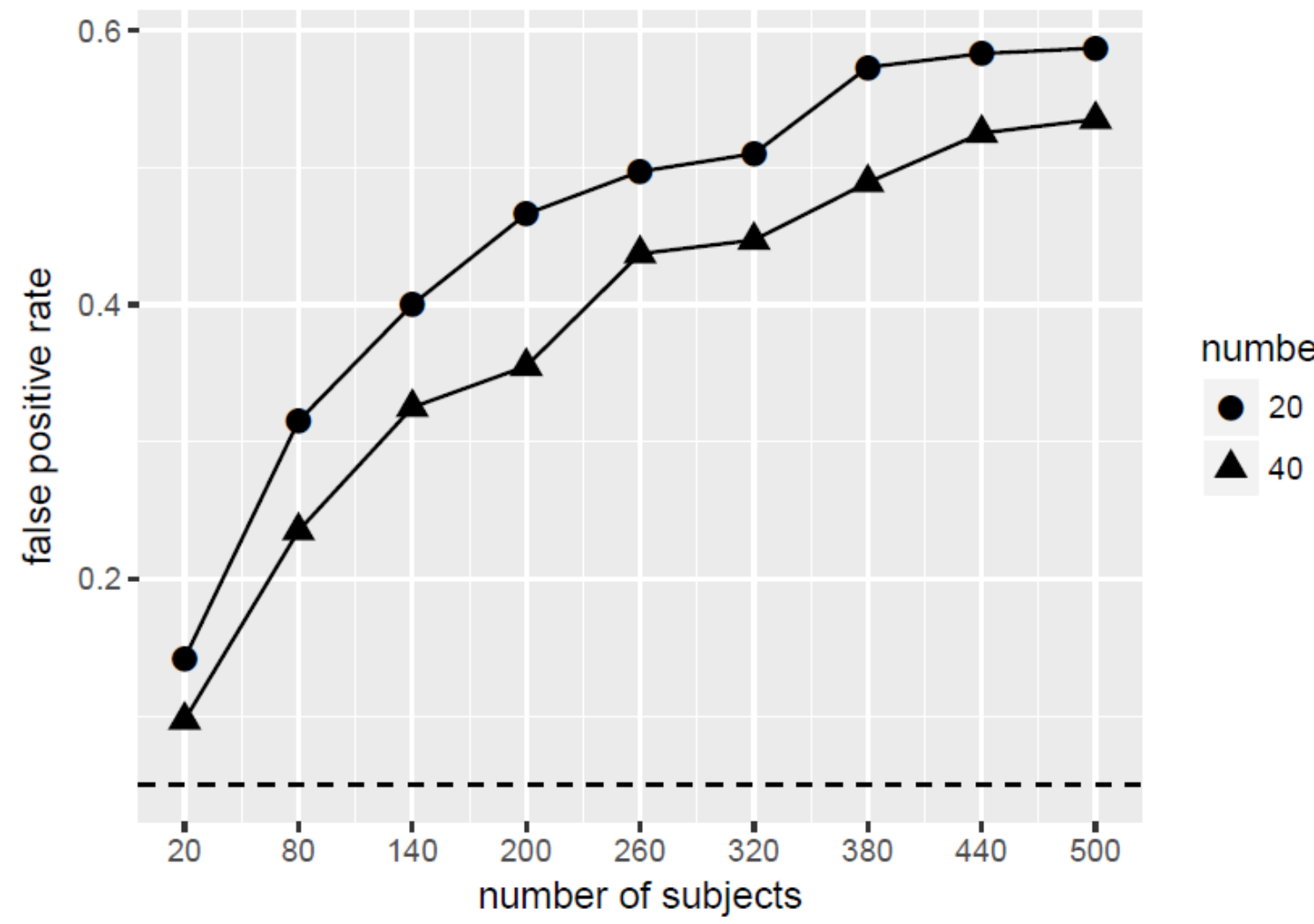

Figure 1. False-positive rates for analyses neglecting stimulus variation as a function of subject sample size (20 to 500 in steps of 60) and stimulus sample size (20 or 40).

This phenomenon also has implications for studies where stimuli or subjects are matched across conditions. In some psycholinguistic studies, for example, researchers take care to match the properties of words (e.g., frequency, imageability, word length) used in different experimental conditions. Upon observing no significant differences between the two sets of stimuli along these dimensions, they will argue that the matching procedure succeeded in creating equivalent sets. But the sets are of course never exactly equivalent, and the significance test that is supposed to demonstrate this is performed on a single instance of each stimulus. However, because each new subject involves a full repetition of the sets of items, any small differences become amplified as new subjects are added, and this noise will be increasingly likely to be mistaken for signal so long as the stimuli are not accounted for in the analysis.

A related case concerns studies in which the same stimuli are used in different conditions, having been also appropriately counterbalanced across conditions. It is a misconception that this exempts the analyst from having to consider items in the analysis. Although a paper by Raaijmakers et al. (1999) seems to suggest this, this misses a critical detail of that paper: their analysis for the withinitem design actually did take item effects into account. ${ }^{2}$ All that repeating items across conditions controls for is the random intercept variation associated with items, ensuring that this variation is not confounded with the factor of interest. However, it ignores the likelihood of treatment variation across items - i.e., that items will vary in the extent to which they show an effect across conditions. For instance, in studies on perspective taking in language comprehension, it is common to manipulate the identity of privileged objects: objects that listeners can see but know that speakers can't see and don't know about. A speaker might ask the listener to e.g., move the tape one space right, and listeners might see, in addition to a "target" tape that is shared with the speaker, a hidden

2 Albeit indirectly, through the proxy of a 'list' effect included in the model. 
object that is also called tape ("competitor" condition) or is called something else such as an apple ("noncompetitor" condition). Listeners are more likely to look at the privileged object when it matches the target, but whatever effect the competitor manipulation produces for this particular set of stimuli, it would differ from the competitor effect for another set (e.g., move the small cup one space right, when there is a large and smaller "target" cup, and the privileged competitor is either an even smaller cup or a toy monkey. Each stimulus has a unique "house effect". If every participant encounters all of the stimuli, then each participant also replicates the house effect for each one, and these idiosyncracies accumulate.

As Clark (1973) noted, there is one situation in which it is legitimate to neglect sampled units in an analysis, and that is when each sampled unit appears only once in the entire sample-i.e., when a sampled unit does not participate in more than one encounter. This approach works because variation associated with the sampled units is fully absorbed in the residual variance (in technical terms, the variation is not identifiable by any statistical model). For instance, if each subject in the sample responds to a unique set of words, then the variance associated with each word is fully accounted for by the model residual, and only a by-subjects analysis is needed.

\section{How to account for sampling variation in a statistical model?}

It is important not only whether a particular source of variation is taken into account in an analysis, but also how this variation is captured by the model. As noted above, following the landmark Baayen et al. (2008) paper on linear mixed effects models, many researchers mistakenly assumed that incorporating sampling units as random intercepts was sufficient for generalization. However, as Barr et al. (2013) pointed out, such random-intercepts-only models are only appropriate when sampling units are nested within levels of experimental factors, and such designs are unusual in psycholinguistics. When sampling units participate in multiple encounters at each level of an experimental factor, this introduces variation that must be accounted for by estimating random slope variation associated with those units. Failure to do so can increase false positive rates dramatically; indeed, Barr et al. (2013) found that for certain designs where a factor was administered within subjects as well as within items, random-intercepts-only models showed worse generalization than a by-subjects analysis alone.

Barr et al. (2013) provide some guidelines for determining what the maximal random effects structure should be for a particular design, and encourage researchers to try to estimate models with maximal random effects. The general idea is that each sampling unit gets a random intercept, and random slopes for any factor for which two conditions are satisfied: (1) the levels of the factor are administered within-unit, and (2) there are multiple observations for each unit at each level of the factor. The researcher should consider whether or not to include random slopes from the point of view of each sampling unit in the study. For instance, a by-subject random slope for factor X may be needed if both criteria are satisfied from the point of view of individual subjects, but a by-item random slope may not be needed if they are not satisfied from the point of view of individual items (e.g., the factor is administered between items). One should also consider whether observations are replicated over individual encounters; it is possible that each participant encounters the same stimulus multiple times during the experiment, and if this is the case, then by-encounter random intercepts and random slopes should also be considered, following the same logic. ${ }^{3}$ For guidance on specifying interaction terms in factorial designs, see Barr (2013).

\section{Conclusion}

The basic claim of this paper is that the target of generalization in psychology and neuroscience has

3 Note that for studies involving person-stimulus encounters, the 'encounter' is what Barr et al. (2013) refer to as the 'subject-by-stimulus interaction'. 
been misidentified as a particular population of subjects, or in some cases, separate populations of subjects and items. I have argued that the proper target of generalization is a population of events involving encounters between sampling units: language users encountering particular types of sentences, female raters encountering particular types of male faces, students encountering particular types of instructors, brains encountering particular types of scenes, potential employers encountering different types of potential employees, birds encountering particular types of songs. The inferential target in such cases is clearly the populations of events of which the sampling units are but constituent elements. This broader perspective clarifies that the need to consider stimuli in one's analysis has very much to do with the fact they are sampled and very little to do with whether the stimuli are words, faces, images, or something else. It also provides a non-technical motivation for why separate analyses for each of the populations involved in the encounter fail to justify claims about the target population of events. Finally, this broader perspective might help researchers appreciate limits on the generality of a study as well as what aspects of a study should be targeted in a replication effort.

Generalizing over encounters presents a number of practical challenges that are beyond the scope of this chapter (for discussion, see Barr et al., 2013; Matuschek et al., 2017; Westfall et al., 2016). While the advent of mixed-effects modeling has made it possible to flexibly model all sources of sampling error within a single framework, in practice, the estimation algorithm is not guaranteed to arrive at a solution, often requiring a reduction in the random effects structure. Although there are conflicting ideas about whether it is prudent to perform significance tests on random slope variance when specifying a model, all experts agree that it is essential to consider associated random slope variance when performing significance tests on fixed effects.

Mixed-effects models are powerful, but they have the critical weakness of requiring explicit estimation of variation even in situations in which such variation is best considered as 'nuisance' variation that is not of theoretical interest. McNeish, Stapleton, and Silverman (2017) criticized the "unnecessary ubiquity" of mixed-effects models in psychology, pointing out that other "populationaverage models' (PAMs) exist, such as Generalized Estimation Equations (Liang \& Zeger, 1986). Such models do not require explicit estimation of such variation and thus analyses are less likely to suffer convergence problems. However, there are currently no PAM approaches that allow for crossed random factors. Until such approaches are developed, linear mixed-effects models remain the best available option.

Psycholinguistics has had much to contribute to the improvement of statistical methods across psychology and neuroscience, as it is currently only the field of psycholinguistics that gets statistical generalization consistently right, although it is only very recently in its historical development that it can claim to do so. It is hoped that by viewing generalization from a more general perspective based on encounters, more researchers will appreciate the critical role that statistical modeling plays in supporting valid, replicable claims about phenomena in psychology and neuroscience.

\section{Author's Note}

Thanks to Gareth Gaskell and Lisa Debruine for comments on an earlier draft of this paper.

\section{References}

Baayen, R. H., Davidson, D. J., \& Bates, D. M. (2008). Mixed-effects modeling with crossed random effects for subjects and items. Journal of Memory and Language, 59, 390-412.

Barr, D. J. (2013). Random effects structure for testing interactions in linear mixed-effects models. Frontiers in Psychology, 4, 328+. 
Barr, D. J., Levy, R., Scheepers, C., \& Tily, H. J. (2013). Random effects structure for confirmatory hypothesis testing: Keep it maximal. Journal of Memory and Language, 68, 255-278.

Bates, D., Mächler, M., Bolker, B. M., \& Walker, S. C. (2015). Fitting linear mixed-effects models using lme4. Journal of Statistical Software, 67, 1-48.

Bedny, M., Aguirre, G. K., \& Thompson-Schill, S. L. (2007). Item analysis in functional magnetic resonance imaging. Neuroimage, 35, 1093-1102.

Clark, H. H. (1973). The language-as-fixed-effect fallacy: A critique of language statistics in psychological research. Journal of Verbal Learning and Verbal Behavior, 12, 335-359.

Cohen, J. (1976). Random means random. Journal of Verbal Learning and Verbal Behavior, 15, 261-262.

Coleman, E. B. (1964). Generalizing to a language population. Psychological Reports, 14, 219-226.

Forster, K., \& Dickinson, R. (1976). More on the language-as-fixed-effect fallacy: Monte Carlo estimates of error rates for F1, F2, F', and min F'. Journal of Verbal Learning and Verbal Behavior, $15,135-142$.

Garrod, S., Fay, N., Lee, J., Oberlander, J., \& MacLeod, T. (2007). Foundations of representation: Where might graphical symbol systems come from? Cognitive Science, 31, 961-987.

Judd, C. M., Westfall, J., \& Kenny, D. A. (2012). Treating stimuli as a random factor in social psychology: a new and comprehensive solution to a pervasive but largely ignored problem. Journal of Personality and Social Psychology, 103, 54-69.

Liang, K.-Y., \& Zeger, S. (1986). Longitudinal data analysis using generalized linear models. Biometrika, 73, 13-22.

Locker, L., Hoffman, L., \& Bovaird, J. (2007). On the use of multilevel modeling as an alternative to items analysis in psycholinguistic research. Behavior Research Methods, 39, 723-730.

MacNell, L., Driscoll, A., \& Hunt, A. N. (2015). What's in a name? Exposing gender bias in student ratings of teaching. Innovative Higher Education, 40, 291-303.

Matuschek, H., Kliegl, R., Vasishth, S., Baayen, H., \& Bates, D. (2017). Balancing Type I error and power in linear mixed models. Journal of Memory and Language, 94, 305-315.

McNeish, D., Stapleton, L. M., \& Silverman, R. D. (2017). On the unnecessary ubiquity of hierarchical linear modeling. Psychological Methods, 22, 114-140.

Pardo, J. S. (2006). On phonetic convergence during conversational interaction. Journal of the Acoustical Society of America, 119, 2382-2393.

Quené, H., \& van den Bergh, H. (2004). On multi-level modeling of data from repeated measures designs: a tutorial. Speech Communication, 43, 103-121.

Raaijmakers, J. G. W., Schrijnemakers, J. M. C., \& Gremmen, F. (1999). How to Deal with "The Language-as-Fixed-Effect Fallacy": Common Misconceptions and Alternative Solutions. Journal of Memory and Language, 41, 416-426.

Schielzeth, H., \& Forstmeier, W. (2009). Conclusions beyond support: overconfident estimates in mixed models. Behavioral Ecology, 20, 416-420.

Smith, J. E. K. (1976). The Assuming-Will-Make-It-So Fallacy. Journal of Verbal Learning and Verbal Behavior, 15, 262-263.

Westfall, J., Judd, C. M., \& Kenny, D. A. (2015). Replicating studies in which samples of participants respond to samples of stimuli. Perspectives on Psychological Science, 10, 390-399. 
Westfall, J., Nichols, T. E., \& Yarkoni, T. (2016). Fixing the stimulus-as-fixed-effect fallacy in task fMRI. Wellcome Open Research, 1, 23. http://doi.org/10.12688/wellcomeopenres.10298.2

Wike, E., \& Church, J. (1976). Comments on Clark's "The language-as-fixed-effect fallacy". Journal of Verbal Learning and Verbal Behavior, 15, 249-255. 